\title{
Few-Mode Field Quantization of Arbitrary Electromagnetic Spectral Densities
}

\author{
Ivan Medina $\oplus^{1,2}$ Francisco J. García-Vidal ${ }^{1,3}$ Antonio I. Fernández-Domínguez ${ }^{1,{ }^{*}}$ and Johannes Feist $\oplus^{1, \dagger}$ \\ ${ }^{1}$ Departamento de Física Teórica de la Materia Condensada and Condensed Matter Physics Center (IFIMAC), \\ Universidad Autónoma de Madrid, E-28049 Madrid, Spain \\ ${ }^{2}$ Centro de Ciências Naturais e Humanas, Universidade Federal do ABC, 09210-170 Santo André, Sao Pãulo, Brazil \\ ${ }^{3}$ Donostia International Physics Center (DIPC), E-20018 Donostia/San Sebastián, Spain
}

(Received 1 August 2020; accepted 11 February 2021; published 3 March 2021)

\begin{abstract}
We develop a framework that provides a few-mode master equation description of the interaction between a single quantum emitter and an arbitrary electromagnetic environment. The field quantization requires only the fitting of the spectral density, obtained through classical electromagnetic simulations, to a model system involving a small number of lossy and interacting modes. We illustrate the power and validity of our approach by describing the population and electric field spatial dynamics in the spontaneous decay of an emitter placed in a complex hybrid plasmonic-photonic structure.
\end{abstract}

DOI: 10.1103/PhysRevLett.126.093601

Motivated by the desire to use nanophotonic devices for quantum optics and quantum technology applications, there is large interest in developing strategies for quantizing electromagnetic (EM) modes in open, dispersive, and absorbing photonic environments, where standard ways of obtaining quantized modes are not valid [1,2]. While macroscopic quantum electrodynamics (QED) provides a framework for such quantization in material structures described by EM constitutive relations [3-9], it describes electromagnetic fields by a continuum of harmonic oscillators, restricting its applicability to cases where they can be treated perturbatively or eliminated by Laplace transform or similar techniques. It is thus desirable to develop tractable but versatile models using only a small number of EM modes. One notable development in this direction is pseudomode theory [10-12]. Work on specific structures has focused on (possibly approximately) obtaining quantized few-mode descriptions for plasmonic (metallic) geometries such as surfaces [13], spheres [14-17], or sphere dimers $[18,19]$.

Within nanophotonics, increasing attention has recently focused on hybrid metallodielectric setups [20-23], aiming to combine the strong field confinement and enhanced light-matter interactions of plasmonic resonances with the long lifetime (large quality factors) of microcavity or photonic crystal modes. EM field quantization is then particularly complex due to the inherent coexistence of modes with very different properties and their mutual

Published by the American Physical Society under the terms of the Creative Commons Attribution 4.0 International license. Further distribution of this work must maintain attribution to the author(s) and the published article's title, journal citation, and DOI. coupling. Quasinormal modes [22,24-26] can be useful to unveil the EM mode structure, but due to their lossy nature, direct quantization remains challenging, and has only been carried out within limited spectral windows $[23,27]$. A complementary technique developed very recently in the context of x-ray quantum optics is based on a partition of the physical space [28].

The interaction of a single emitter with an arbitrary EM environment can be fully described by the spectral density $J(\omega)$, obtained from classical EM calculations, which encodes the EM mode density and emitter-mode coupling. In this Letter, we present a simple and easily implementable framework for obtaining a few-mode quantum description of any given spectral density. Starting from macroscopic QED, we construct a model system consisting of a discrete number of interacting modes coupled to independent flat background baths; see Fig. 1. Fano diagonalization $[29,30]$ then provides a compact form for the model spectral density, $J_{\text {mod }}(\omega)$, which can be fitted to any level of accuracy to $J(\omega)$. We illustrate the power and validity of this procedure in a hybrid structure comprising a plasmonic nanocavity and a highrefractive-index microresonator. The few-mode model retains the information about the full environment, as we show by accurate calculations of far- and near-field observables.

For a single emitter, the macroscopic QED Hamiltonian can be written as ( $\hbar=1$ here and later) $[16,31-33]$

$$
H_{\mathrm{f}}=H_{e}+\int_{0}^{\infty} d \omega\left[\omega a_{\omega}^{\dagger} a_{\omega}+D_{e} g(\omega)\left(a_{\omega}+a_{\omega}^{\dagger}\right)\right],
$$

where $H_{e}$ is the bare emitter Hamiltonian and $D_{e}$ is its dipole operator. The bosonic annihilation operators $a_{\omega}$, with $\left[a_{\omega}, a_{\omega^{\prime}}^{\dagger}\right]=\delta\left(\omega-\omega^{\prime}\right)$, describe "emitter-centered" 


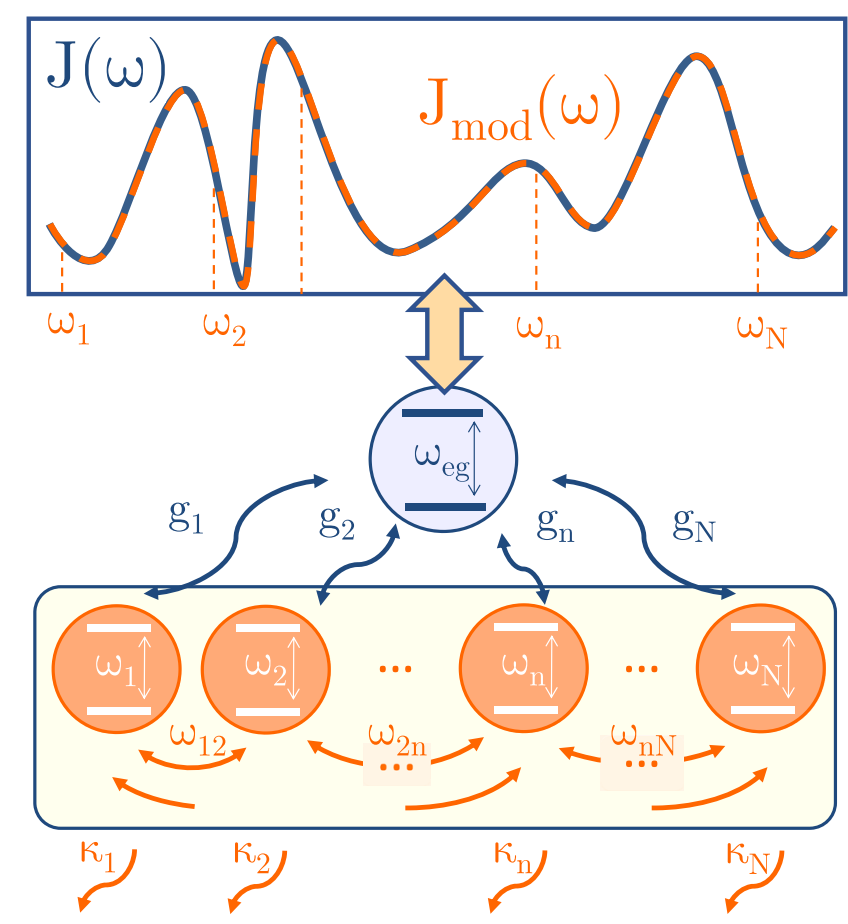

FIG. 1. Sketch of the model and quantization approach. Fitting an original spectral density, $J(\omega)$ (usually obtained by numerical simulations) to a few-mode model $J_{\text {mod }}(\omega)$, Eq. (5), provides quantized EM modes described by the master equation Eq. (4).

EM modes at frequency $\omega$ [34] and correspond to the "true modes" of Refs. [11,12]. The light-matter coupling is

$$
g(\omega)=\sqrt{\frac{\omega^{2}}{\pi \epsilon_{0} c^{2}} \vec{n} \cdot \operatorname{Im}\left\{\mathbf{G}\left(\vec{r}_{e}, \vec{r}_{e}, \omega\right)\right\} \cdot \vec{n},}
$$

with $\vec{r}_{e}$ the emitter position, $\vec{n}$ the orientation of its dipole moment (for simplicity, we assume that all relevant transitions are oriented identically), and $\mathbf{G}\left(\vec{r}, \vec{r}^{\prime}, \omega\right)$ the classical dyadic Green's function. This is directly related to the spectral density, $J(\omega)=\mu^{2} g(\omega)^{2}$ for transition dipole moment $\mu$ [35].

Without approximations, the treatment of an EM continuum, as present in Eq. (1), is only possible with advanced and costly computational techniques, such as tensor network approaches [36,37]. Our goal is thus to construct an equivalent system described by dynamical equations that can be solved easily. Our model (sketched in Fig. 1) consists of $N$ interacting EM modes with ladder operators $a_{i}, a_{i}^{\dagger}$. Each is linearly coupled to the quantum emitter, and also to an independent Markovian (spectrally flat) background bath. The resulting Hamiltonian is $\mathcal{H}=H_{S}+H_{B}$, with

$$
H_{S}=H_{e}+\sum_{i, j=1}^{N} \omega_{i j} a_{i}^{\dagger} a_{j}+D_{e} \sum_{i=1}^{N} g_{i}\left(a_{i}+a_{i}^{\dagger}\right),
$$

$H_{B}=\sum_{i=1}^{N} \int\left[\Omega b_{i, \Omega}^{\dagger} b_{i, \Omega}+\sqrt{\frac{\kappa_{i}}{2 \pi}}\left(b_{i, \Omega}^{\dagger} a_{i}+b_{i, \Omega} a_{i}^{\dagger}\right)\right] d \Omega$.

$H_{S}$ is the system (emitter + discrete modes) Hamiltonian, where the real symmetric matrix $\omega_{i j}$ describes the mode energies and their interactions, and the real positive vector $g_{i}$ describes their coupling to the emitter. The bath Hamiltonian $H_{B}$ contains both the continuous bath modes, described by bosonic operators fulfilling $\left[b_{i^{\prime}, \Omega^{\prime}}, b_{i, \Omega}^{\dagger}\right]=\delta_{i, i^{\prime}} \delta\left(\Omega-\Omega^{\prime}\right)$, and their coupling to the system, characterized by the rates $\kappa_{i}$. Note that the linear character of the light-matter coupling in Eq. (3) originates directly from Eq. (1).

The power of our approach lies in the fact that the Hamiltonian above can be analytically treated in two different ways: First, the Markov approximation for the completely flat background baths $H_{B}$ is exact and the dynamics described by $\mathcal{H}$ is identically reproduced, as proven recently [38], by the master equation

$$
\dot{\rho}=-i\left[H_{S}, \rho\right]+\sum_{i} \kappa_{i} L_{a_{i}}[\rho]
$$

where $\rho$ is the system density matrix and $L_{O}[\rho]=O \rho O^{\dagger}-$ $\frac{1}{2}\left\{O^{\dagger} O, \rho\right\}$ is a standard Lindblad dissipator. For $N=2$, Eq. (4) recovers the ad hoc master equation model in Ref. [39] describing a single photonic Fano resonance.

Second, the system of $N$ interacting modes and continua can be diagonalized by adapting Fano diagonalization, originally developed for autoionizing states of atomic systems [29], and related to quasimode and pseudomode theory $[11,12]$ in this context. This strategy allows us to obtain a simple, closed expression for $J_{\bmod }(\omega)$. This is achieved by solving the Lippmann-Schwinger equations to obtain the $N$ eigenmodes of the model at each frequency $\omega$. Subsequently forming their unique linear superposition (the "bright" or "emitter-centered" mode) coupling to the emitter leads to a compact expression for the model spectral density (for details, see the Supplemental Material [40]):

$$
J_{\text {mod }}(\omega)=\frac{\mu^{2}}{\pi} \operatorname{Im}\left\{\vec{g}^{T} \frac{1}{\tilde{\mathbf{H}}-\omega} \vec{g}\right\} .
$$

Here, $\vec{g}^{T}=\left(g_{1}, g_{2}, \ldots, g_{N}\right)$ is an $N$-element vector. The $N \times N$ matrix $\tilde{\mathbf{H}}$ has entries $\tilde{\mathbf{H}}_{i j}=\omega_{i j}-\frac{i}{2} \kappa_{i} \delta_{i j}$, where Lamb shifts due to the coupling with the baths have been absorbed into the mode frequencies $\omega_{i i}$ [as also implicitly done in Eq. (4)]. As required for a spectral density, $J_{\bmod }(\omega)$ is non-negative for all $\omega$ [40].

The last step in our approach consists in using Eq. (5) to fit $J(\omega)$ for a given EM environment to parametrize Eq. (4) for that system. Although the number of unknowns in $J_{\text {mod }}(\omega)$ is relatively large $\left(N^{2}+2 N\right.$ real numbers for $\omega_{i j}$, 

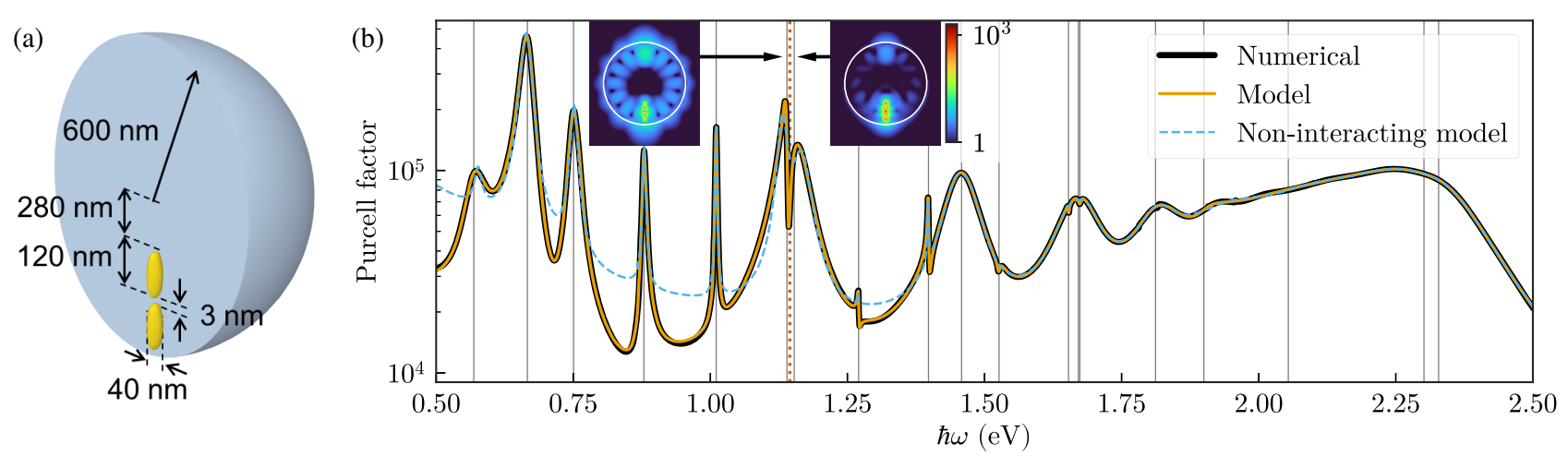

FIG. 2. (a) Sketch of the model system consisting of a silver dimer nanoantenna embedded in a dielectric microsphere. (b) Purcell factor $J(\omega) / J_{0}(\omega)$ for the system (thick black line), for the fitted model described by Eq. (5) with 20 modes (orange line), and for a model without interactions with the same number of modes (dashed light blue line). Thin gray lines indicate the energy positions of the eigenstates of $\tilde{\mathbf{H}}$ for the fitted interacting system. The red dotted line indicates the frequency of the emitter used in Fig. 3 and Fig. 4 . The insets show the electric field distributions for the two modes indicated by the arrows (see main text for details).

$\kappa_{i}$, and $\left.g_{i}\right)$, the fit procedure turns out to be stable even for large numbers of modes $(N=20$ in the example below). A similar procedure based on the fitting of the bath correlation function has been reported as problematic recently [43]. For noninteracting modes, $\omega_{i j}=\omega_{i} \delta_{i j}$, Eq. (5) simplifies to a sum of Lorentzians of the form $J_{\bmod }(\omega)=\sum_{i}\left(\mu^{2} g_{i}^{2} / \pi\right)\left\{\left[\kappa_{i} / 2\right] /\left[\left(\omega-\omega_{i}\right)^{2}+\kappa_{i}^{2} / 4\right]\right\}$, reproducing the well-known relation between Lorentzian spectral densities and lossy modes [10,44] that has been widely used to quantize simpler EM environments such as plasmonic cavities in the quasistatic approximation $[13,15,17,18]$. The introduction of interactions allows significantly more freedom in fitting $J_{\text {mod }}(\omega)$, and, in particular, allows for the representation of interference effects indicated by Fano-like line shapes. In the Supplemental Material [40], explicit expressions of Eq. (5) for up to 4 interacting modes are presented, as well as their fitting to $J(\omega)$ in two recent studies $[22,23]$. In the following, we illustrate the power of our approach by considering a hybrid nanophotonic structure with a significantly more complex spectral density.

Figure 2(a) shows the system under study: a $600 \mathrm{~nm}$ radius $\mathrm{GaP}[45]$ microsphere $\left(\varepsilon_{\mathrm{sph}}=9\right)$ embedding two $120 \mathrm{~nm}$ long silver nanorods (with permittivity taken from Ref. [46]) separated by a $3 \mathrm{~nm}$ gap, substantially displaced from the center of the sphere. The microsphere by itself supports many long-lived and delocalized Mie resonances, while the plasmonic dimer sustains confined surface plasmons with strongly subwavelength effective volumes [47]. The interaction between these different modes leads to a complex EM spectrum, shown in Fig. 2(b) through the Purcell factor $P(\omega)=J(\omega) / J_{0}(\omega)$, with $J_{0}(\omega)=$ $\left(\omega^{3} \mu^{2} / 6 \pi^{2} \hbar \varepsilon_{0} c^{3}\right)$ the spectral density in free space, for an emitter located in the center of the nanorods. The thick black line plots classical EM simulations performed with the Maxwell equation solver of COMSOL Multiphysics. This Purcell factor, and the corresponding $J(\omega)$, presents a large number of maxima, with several Fano-like profiles that indicate interference effects as typical for hybrid metallodielectric systems [22,23].

In order to stably fit $J_{\bmod }(\omega)$ to $J(\omega)$ despite the large number of parameters, we first fit the noninteracting model (where $\omega_{i j}=\omega_{i} \delta_{i j}$ ), which converges rapidly by using the positions, curvatures, and amplitudes of the local maxima in $J(\omega)$ to obtain initial guesses for the frequencies $\omega_{i}$, loss rates $\kappa_{i}$, and coupling strengths $g_{i}$. This gives a good fit for many of the peaks [dashed light blue line in Fig. 2(b)], but strongly overestimates the background at lower frequencies, and fails to reproduce the Fano-like asymmetric profiles due to hybridization between sphere and dimer modes. Using the noninteracting model for the initial values leads to rapid convergence for the full model with interactions. The resulting spectrum [orange line in Fig. 2(b)] is in almost perfect agreement with the numerical result over the full frequency range. We remark that $J_{\text {mod }}(\omega)$ involves only 20 interacting modes, while numerical calculations (not shown here) indicate that the number of quasinormal modes in the broad frequency range spanned in Fig. 2 is significantly larger. Furthermore, a similarly accurate fit using noninteracting modes would require a significantly larger number of modes.

The thin gray lines in Fig. 2(b) indicate the (real part) of the eigenenergies of the matrix $\tilde{\mathbf{H}}$. These correspond to the complex resonances (poles) of $J(\omega)$ [12]. The insets show the field profiles of two of these modes projected into the space of the modes $a_{\omega}$ of Eq. (1), obtained by identifying the eigenstates of the model [40] with the original modes $a_{\omega}$ and using their field profiles given in Eq. (7) below. This shows the clearly different character of the two modes that interfere to produce a nontrivial spectral density feature at $\hbar \omega \approx 1.15 \mathrm{eV}$.

We next demonstrate that the model system indeed gives a faithful representation of the EM environment, i.e., that the dynamics with the model and with the original 


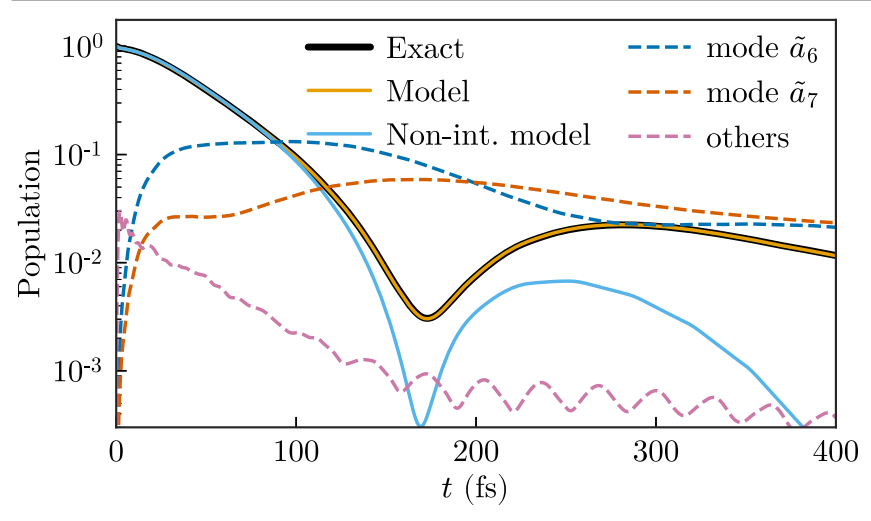

FIG. 3. Emitter and mode dynamics for the spontaneous emission problem. Solid lines show the emitter excited-state population $\left\langle\sigma^{+} \sigma^{-}\right\rangle(t)$ for the exact calculation (black) and fitted models with (orange) and without (light blue) interactions. Dashed lines indicate the populations $\left\langle\tilde{a}_{\alpha}^{\dagger} \tilde{a}_{\alpha}\right\rangle(t)$ of modes $\alpha=$ 6 (blue) and $\alpha=7$ (red) (see text for details), and the sum over all other mode populations (magenta).

Hamiltonian are equivalent. To do so, we treat the canonical spontaneous emission (Wigner-Weisskopf) problem for a two-level emitter initially in its excited state [48], with $H_{e}=\omega_{e g} \sigma^{+} \sigma^{-}$and $D_{e}=\mu\left(\sigma^{+}+\sigma^{-}\right)$, where $\sigma^{ \pm}$are Pauli matrices. The emitter parameters are chosen to represent InAs/InGaAs quantum dots [49], with transition energy $\hbar \omega_{e g}=1.145 \mathrm{eV}$ [dotted red line in Fig. 2(b)], and transition dipole moment $\mu=0.55 \mathrm{enm}$.

Since in this problem, there is at most one excitation in the system (either in the emitter or in one of the EM modes), it can be solved easily for arbitrary spectral densities [44]. The exact excited-state population $\left\langle\sigma^{+} \sigma^{-}\right\rangle(t)$ obtained through this approach is shown in Fig. 3 (thick black line). The clearly reversible dynamics proves the non-Markovian character of the interaction between the emitter and its EM environment. The population obtained from the full model (orange line) reproduces the exact results perfectly, while the noninteracting model (light blue) fails to do so and shows significant deviations after about 100 fs. In the Supplemental Material, we additionally show that perfect agreement is also obtained when considering a four-level emitter that decays by two-photon emission [40]. The solution of the model master equations was calculated using QuTiP [50].

In order to gain further insight into the relevance and meaning of the discrete modes obtained in our fit, we also show the populations of the modes $\tilde{a}_{\alpha}$ obtained by diagonalizing the real symmetric matrix $\omega_{i j}$. This corresponds to an orthogonal transformation of the modes $a_{i}$, with $\tilde{a}_{\alpha}=\sum_{i} V_{\alpha i} a_{i}$. The dashed lines in Fig. 3 show the populations of modes $\tilde{a}_{6}$ and $\tilde{a}_{7}$, the modes closest to resonance to the emitter frequency, $\tilde{\omega}_{6}=1.129$ and $\tilde{\omega}_{7}=$ $1.149 \mathrm{eV}$ (see Fig. 2). The sum over all other mode populations, shown as a dashed magenta line in Fig. 3, remains small during the whole propagation. This demonstrates that our approach also allows the identification of the relevant modes in the dynamics. However, we note that the emitter dynamics are not correctly reproduced if the other modes are dropped from the simulation. In particular, the broad plasmonic pseudomode peak extending from $\approx 1.85$ to $2.5 \mathrm{eV}$, which contains the effect of many higherorder multipole modes, induces a significant energy shift on the emitter [15], which is not captured correctly when these modes are removed from the simulation.

Finally, we show that although the model system is written in terms of discrete lossy modes, it retains the full information about the EM near and far field. The electric field operator for the modes $a_{\omega}$ in Eq. (1) can be written as [34]

$$
\vec{E}^{(+)}(\vec{r})=\int_{0}^{\infty} \overrightarrow{\mathcal{E}}(\vec{r}, \omega) a_{\omega} d \omega,
$$

where the field mode profile is given by

$$
\overrightarrow{\mathcal{E}}(\vec{r}, \omega)=\frac{\hbar \omega^{2}}{\pi \epsilon_{0} c^{2} g(\omega)} \operatorname{Im}\left\{\mathbf{G}\left(\vec{r}, \vec{r}_{e}, \omega\right)\right\} \cdot \vec{n} .
$$

Formally integrating the Heisenberg equations of motion for $a_{\omega}(t)$ yields

$$
a_{\omega}(t)=a_{\omega}(0) e^{-i \omega t}-i g(\omega) \int_{0}^{t} D_{e}\left(t^{\prime}\right) e^{-i \omega\left(t-t^{\prime}\right)} d t^{\prime} .
$$

Inserting into Eq. (6) and defining the temporal kernel

$\vec{K}(\vec{r}, \tau)=\frac{\hbar}{\pi \epsilon_{0} c^{2}} \int_{0}^{\infty} \omega^{2} \operatorname{Im}\left\{\mathbf{G}\left(\vec{r}, \vec{r}_{e}, \omega\right)\right\} \cdot \vec{n} e^{i \omega \tau} d \omega$

gives compact expressions for, e.g., the electric field intensity

$$
\begin{aligned}
\left\langle\vec{E}^{(-)} \cdot \vec{E}^{(+)}\right\rangle= & \int_{0}^{t} d t^{\prime} \int_{0}^{t} d t^{\prime \prime}\left\langle D_{e}\left(t^{\prime}\right) D_{e}\left(t^{\prime \prime}\right)\right\rangle \\
& \times \vec{K}\left(\vec{r}, t-t^{\prime}\right) \vec{K}^{*}\left(\vec{r}, t-t^{\prime \prime}\right),
\end{aligned}
$$

where we have assumed that the field is initially in the vacuum state.

Equation (10) enables the calculation of the field intensity anywhere in space through the emitter correlation functions, easily obtained from Eq. (4). This is displayed for the spontaneous emission case of Fig. 3 in Fig. 4. Panel (a) shows the temporal dependence of the electric field intensity at various points in space, with locations indicated by numbered white circles in panel (c). The "direct" calculation (not shown) of the field intensities, possible for the Wigner-Weisskopf problem, gives essentially perfect agreement with the results obtained through Eq. (10). The intensities at each point are normalized to their 

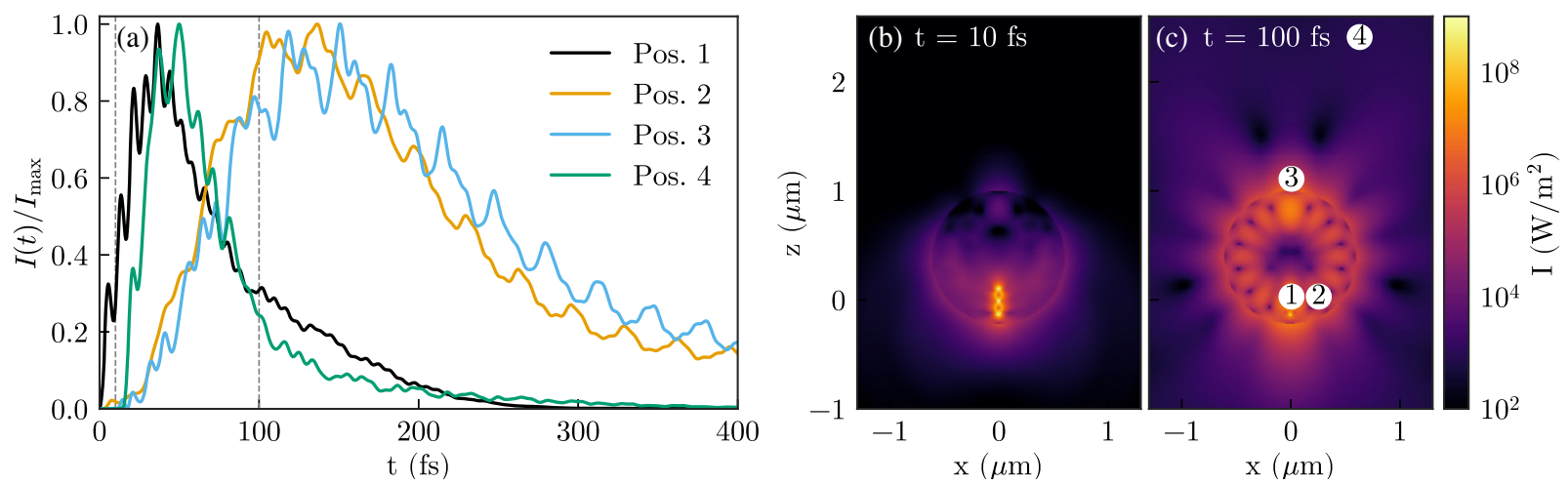

FIG. 4. Electric field intensity $\left\langle\vec{E}^{(-)}(\vec{r}) \cdot \vec{E}^{(+)}(\vec{r})\right\rangle$ for the initially excited emitter as in Fig. 3. (a) Field intensity at four points, shown by numbered white circles in panel (c), normalized to their maximum values $I_{\text {max }}$, given by $2.0 \times 10^{8} \mathrm{~W} / \mathrm{m}^{2}, 1.8 \times 10^{6} \mathrm{~W} / \mathrm{m}^{2}$, $1.1 \times 10^{6} \mathrm{~W} / \mathrm{m}^{2}$, and $4.0 \times 10^{3} \mathrm{~W} / \mathrm{m}^{2}$, respectively. (b) Field intensity distribution in the $x-z$ plane at time $t=10 \mathrm{fs}$, and (c) at $t=100 \mathrm{fs}$.

maximum value (see figure caption). Panels (b) and (c) show snapshots of the field intensity profile in space at $t=10$ and $t=100 \mathrm{fs}$, respectively. A movie showing the field evolution is available in the Supplemental Material [40]. We note explicitly that in this spontaneous emission problem, there is no coherent field, $\left\langle\vec{E}^{(+)}+\vec{E}^{(-)}\right\rangle=0$, and the field dynamics are only visible in the intensity. Interestingly, the dynamics at points next to the emitter, (position 1), and in the far-field $1.5 \mu \mathrm{m}$ away from the emitter, (position 4), are quite similar, reaching their maximum value within tens of femtoseconds and then decaying rapidly. In contrast, points (position 2) and (position 3) inside and just outside the dielectric sphere (but at some distance to the nanorod dimer) show a much slower buildup and decay of the field intensity in time. Comparison with Fig. 3 reveals that modes 6 and 7 give the largest contribution to the field intensity at positions 2 and 3 , while the initial fast decay is due to the contribution of other modes and leads to an intense initial pulse radiated from the system (position 4).

To conclude, we have presented a simple and insightful procedure to quantize the electromagnetic field in arbitrary nanophotonic systems. Our approach is based on fitting a model spectral density, obtained through Fano diagonalization and involving only a small number of lossy and interacting electromagnetic modes, to the full spectral density obtained from Maxwell's equations. This makes it possible to construct and parametrize a few-mode master equation accurately describing the interaction of a quantum emitter with the original EM environment. We have illustrated the power and validity of our ideas by calculating the population dynamics and near- and far-field intensity for an emitter placed within a hybrid structure comprising a dielectric microresonator and a plasmonic cavity. Our findings offer a versatile and easily implementable framework for the theoretical description of quantum nano-optical phenomena with dyadic Green's function calculations as the single input.
The authors thank Diego Martín-Cano for interesting discussions and sharing their data. This work has been funded by the European Research Council through Grant ERC-2016-StG-714870 and by the Spanish Ministry for Science, Innovation, and Universities-Agencia Estatal de Investigación through grants RTI2018-099737-B-I00, PCI2018-093145 (through the QuantERA program of the European Commission), and MDM-2014-0377 (through the María de Maeztu program for Units of Excellence in R\&D). It was also supported by a Leonardo Grant for Researchers and Cultural Creators, BBVA Foundation. I. M. thanks the nanophotonics group for the warm hospitality during his stay at UAM, and acknowledges funding from the Coordenação de Aperfeiçoamento de Pessoal de Nível Superior-Brazil (CAPES) through Grant 88887.368031/2019-00.

*a.fernandez-dominguez@uam.es johannes.feist@uam.es

[1] A. F. Koenderink, On the use of Purcell factors for plasmon antennas, Opt. Lett. 35, 4208 (2010).

[2] P. T. Kristensen, C. Van Vlack, and S. Hughes, Generalized effective mode volume for leaky optical cavities, Opt. Lett. 37, 1649 (2012).

[3] U. Fano, Atomic theory of electromagnetic interactions in dense materials, Phys. Rev. 103, 1202 (1956).

[4] B. Huttner and S. M. Barnett, Quantization of the electromagnetic field in dielectrics, Phys. Rev. A 46, 4306 (1992).

[5] S. Scheel, L. Knöll, and D.-G. Welsch, QED commutation relations for inhomogeneous Kramers-Kronig dielectrics, Phys. Rev. A 58, 700 (1998).

[6] L. Knöll, S. Scheel, and D.-G. Welsch, QED in dispersing and absorbing media, in Coherence and Statistics of Photons and Atoms, edited by J. Peřina (WILEY-VCH Verlag, New York, 2001) [arXiv:quant-ph/0006121].

[7] S. Scheel and S.Y. Buhmann, Macroscopic quantum electrodynamics-Concepts and applications, Acta Phys. Slovaca 58, 675 (2008). 
[8] S. Y. Buhmann, Dispersion Forces I, Springer Tracts in Modern Physics Vol. 247 (Springer Berlin Heidelberg, Berlin, Heidelberg, 2012), http://dx.doi.org/10.1007/9783-642-32484-0.

[9] S. Y. Buhmann, Dispersion Forces II, Springer Tracts in Modern Physics Vol. 248 (Springer Berlin Heidelberg, Berlin, Heidelberg, 2012), http://dx.doi.org/10.1007/9783-642-32466-6.

[10] A. Imamoğlu, Stochastic wave-function approach to nonMarkovian systems, Phys. Rev. A 50, 3650 (1994).

[11] B. M. Garraway, Decay of an atom coupled strongly to a reservoir, Phys. Rev. A 55, 4636 (1997); B. M. Garraway, Nonperturbative decay of an atomic system in a cavity, Phys. Rev. A 55, 2290 (1997).

[12] B. J. Dalton, S. M. Barnett, and B. M. Garraway, Theory of pseudomodes in quantum optical processes, Phys. Rev. A 64, 053813 (2001).

[13] A. González-Tudela, P. A. Huidobro, L. Martín-Moreno, C. Tejedor, and F. J. García-Vidal, Reversible dynamics of single quantum emitters near metal-dielectric interfaces, Phys. Rev. B 89, 041402(R) (2014).

[14] E. Waks and D. Sridharan, Cavity QED treatment of interactions between a metal nanoparticle and a dipole emitter, Phys. Rev. A 82, 043845 (2010).

[15] A. Delga, J. Feist, J. Bravo-Abad, and F. J. Garcia-Vidal, Quantum Emitters Near a Metal Nanoparticle: Strong Coupling and Quenching, Phys. Rev. Lett. 112, 253601 (2014).

[16] B. Rousseaux, D. Dzsotjan, G. Colas des Francs, H. R. Jauslin, C. Couteau, and S. Guérin, Adiabatic passage mediated by plasmons: A route towards a decoherence-free quantum plasmonic platform, Phys. Rev. B 93, 045422 (2016).

[17] H. Varguet, B. Rousseaux, D. Dzsotjan, H. R. Jauslin, S. Guérin, and G. Colas des Francs, Non-Hermitian Hamiltonian description for quantum plasmonics: From dissipative dressed atom picture to Fano states, J. Phys. B 52, 055404 (2019).

[18] R.-Q. Li, D. Hernángomez-Pérez, F. J. García-Vidal, and A. I. Fernández-Domínguez, Transformation Optics Approach to Plasmon-Exciton Strong Coupling in Nanocavities, Phys. Rev. Lett. 117, 107401 (2016).

[19] A. Cuartero-González and A. I. Fernández-Domínguez, Light-forbidden transitions in plasmon-emitter interactions beyond the weak coupling regime, ACS Photonics 5, 3415 (2018).

[20] H. M. Doeleman, E. Verhagen, and A. F. Koenderink, Antenna-cavity hybrids: Matching polar opposites for purcell enhancements at any linewidth, ACS Photonics 3, 1943 (2016).

[21] P. Peng, Y.-C. Liu, D. Xu, Q.-T. Cao, G. Lu, Q. Gong, and Y.-F. Xiao, Enhancing Coherent Light-Matter Interactions through Microcavity-Engineered Plasmonic Resonances, Phys. Rev. Lett. 119, 233901 (2017).

[22] B. Gurlek, V. Sandoghdar, and D. Martín-Cano, Manipulation of quenching in nanoantenna-emitter systems enabled by external detuned cavities: A path to enhance strong-coupling, ACS Photonics 5, 456 (2018).

[23] S. Franke, S. Hughes, M. K. Dezfouli, P. T. Kristensen, K. Busch, A. Knorr, and M. Richter, Quantization of Quasinormal Modes for Open Cavities and Plasmonic
Cavity Quantum Electrodynamics, Phys. Rev. Lett. 122, 213901 (2019).

[24] E. S. C. Ching, P. T. Leung, A. Maassen van den Brink, W. M. Suen, S. S. Tong, and K. Young, Quasinormal-mode expansion for waves in open systems, Rev. Mod. Phys. 70, 1545 (1998).

[25] P. T. Kristensen and S. Hughes, Modes and mode volumes of leaky optical cavities and plasmonic nanoresonators, ACS Photonics 1, 2 (2014).

[26] P. Lalanne, W. Yan, K. Vynck, C. Sauvan, and J.-P. Hugonin, Light interaction with photonic and plasmonic resonances, Laser Photonics Rev. 12, 1700113 (2018).

[27] S. Hughes, M. Richter, and A. Knorr, Quantized pseudomodes for plasmonic cavity QED, Opt. Lett. 43, 1834 (2018).

[28] D. Lentrodt and J. Evers, Ab Initio Few-Mode Theory for Quantum Potential Scattering Problems, Phys. Rev. X 10, 011008 (2020).

[29] U. Fano, Effects of configuration interaction on intensities and phase shifts, Phys. Rev. 124, 1866 (1961).

[30] S. Glutsch, Optical absorption of the Fano model: General case of many resonances and many continua, Phys. Rev. B 66, 075310 (2002).

[31] S. Y. Buhmann and D.-G. Welsch, Casimir-Polder forces on excited atoms in the strong atom-field coupling regime, Phys. Rev. A 77, 012110 (2008).

[32] T. Hümmer, F. J. García-Vidal, L. Martín-Moreno, and D. Zueco, Weak and strong coupling regimes in plasmonic QED, Phys. Rev. B 87, 115419 (2013).

[33] M. Sánchez-Barquilla, R. E. F. Silva, and J. Feist, Cumulant Expansion for the treatment of light-matter interactions in arbitrary material structures, J. Chem. Phys. 152, 034108 (2020).

[34] J. Feist, A. I. Fernández-Domínguez, and F. J. García-Vidal, Macroscopic QED for quantum nanophotonics: Emittercentered modes as a minimal basis for multiemitter problems, Nanophotonics 10, 477 (2020).

[35] L. Novotny and B. Hecht, Principles of Nano-Optics, 2nd ed. (Cambridge University Press, Cambridge, England, 2012).

[36] J. del Pino, F. A. Y. N. Schröder, A. W. Chin, J. Feist, and F. J. Garcia-Vidal, Tensor Network Simulation of NonMarkovian Dynamics in Organic Polaritons, Phys. Rev. Lett. 121, 227401 (2018).

[37] D. Zhao, R. E. F. Silva, C. Climent, J. Feist, A. I. FernándezDomínguez, and F. J. García-Vidal, Impact of vibrational modes in the plasmonic Purcell effect of organic molecules, ACS Photonics 7, 3369 (2020).

[38] D. Tamascelli, A. Smirne, S. F. Huelga, and M. B. Plenio, Nonperturbative Treatment of Non-Markovian Dynamics of Open Quantum Systems, Phys. Rev. Lett. 120, 030402 (2018).

[39] E. V. Denning, J. Iles-Smith, and J. Mork, Quantum lightmatter interaction and controlled phonon scattering in a photonic Fano cavity, Phys. Rev. B 100, 214306 (2019).

[40] See Supplemental Material at http://link.aps.org/ supplemental/10.1103/PhysRevLett.126.093601 which contains Refs. [41,42], for the explicit expression for the eigenmodes of the model and the proof of the non-negative character of $J_{\bmod }(\omega)$, the dynamics of a two-photon emitter, 
and the form of the spectral density for up to 4 modes and the fit to the spectral densities in Refs. [22,23].

[41] S. Y. Buhmann, D. T. Butcher, and S. Scheel, Macroscopic quantum electrodynamics in nonlocal and nonreciprocal media, New J. Phys. 14, 083034 (2012).

[42] M. Kamandar Dezfouli and S. Hughes, Quantum optics model of surface-enhanced raman spectroscopy for arbitrarily shaped plasmonic resonators, ACS Photonics 4, 1245 (2017).

[43] F. Mascherpa, A. Smirne, A. D. Somoza, P. FernándezAcebal, S. Donadi, D. Tamascelli, S. F. Huelga, and M. B. Plenio, Optimized auxiliary oscillators for the simulation of general open quantum systems, Phys. Rev. A 101, 052108 (2020).

[44] G. Grynberg, A. Aspect, C. Fabre, and C. Cohen-Tannoudji, Introduction to Quantum Optics: From the Semi-Classical Approach to Quantized Light (Cambridge University Press, Cambridge, England, 2010).

[45] J. Cambiasso, G. Grinblat, Y. Li, A. Rakovich, E. Cortés, and S.A. Maier, Bridging the gap between dielectric nanophotonics and the visible regime with effectively lossless gallium phosphide antennas, Nano Lett. 17, 1219 (2017).

[46] A. D. Rakić, A. B. Djurišić, J. M. Elazar, and M. L. Majewski, Optical properties of metallic films for verticalcavity optoelectronic devices, Appl. Opt. 37, 5271 (1998).

[47] J. J. Baumberg, J. Aizpurua, M. H. Mikkelsen, and D. R. Smith, Extreme nanophotonics from ultrathin metallic gaps, Nat. Mater. 18, 668 (2019).

[48] V. Weisskopf and E. Wigner, Über Die Natürliche Linienbreite in Der Strahlung Des Harmonischen Oszillators, Z. Für Phys. Hadrons Nucl. 65, 18 (1930).

[49] P. G. Eliseev, H. Li, A. Stintz, G. T. Liu, T. C. Newell, K. J. Malloy, and L. F. Lester, Transition dipole moment of InAs/InGaAs Quantum dots from experiments on ultralowthreshold laser diodes, Appl. Phys. Lett. 77, 262 (2000).

[50] J. R. Johansson, P. D. Nation, and F. Nori, QuTiP: An opensource PYTHON framework for the dynamics of open quantum systems, Comput. Phys. Commun. 183, 1760 (2012); J. R. Johansson, P. D. Nation, and F. Nori, QuTiP 2: A PYTHON framework for the dynamics of open quantum systems, Comput. Phys. Commun. 184, 1234 (2013). 